\title{
Whispers in Bedlam: Detecting the Dirac metal at a surface of a topological insulator by means of weak localization
}

Electrically tunable surface-to-bulk coherent coupling in topological insulator thin films. arXiv:1104.1404 (2011)

Authors: H. Steinberg, J.-B. Laloö, V. Fatemi, J. S. Moodera, P. Jarillo-Herrero

and

Tunable surface conductivity in $\mathrm{Bi}_{2} \mathrm{Se}_{3}$ revealed in diffusive electron transport

Phys. Rev. B 83, 241304/1-4 (2011)

Authors: J. Chen, X. Y. He, K. H. Wu, Z. Q. Ji, L. Lu, J. R. Shi, J. H. Smet, and Y. Q. Li

\section{Recommended with a Commentary by Leonid Glazman, Yale University}

The stunning theoretical discovery of 3D topological insulators [1-3] and the associated concrete material suggestions [4-6] touched off an avalanche of experiments aimed at detecting the signature behavior of these unconventional solids. The band structure characteristic for 3D topological insulators was seen in ARPES spectra [7]. Local STM probes also indicated the characteristic suppression of backscattering off the surface imperfections [8, 9]. However, the most desired observation of the hallmark metallic behavior of the dc conduction confined to the surface layer of a 3D topological insulator remains elusive. The main problem is the conduction through the bulk: in reality, these exotic insulators are narrow-gap semiconductors with the gap width in the ballpark of $0.3 \mathrm{eV}$. Apart from thermal excitation, the bulk carriers are provided by the crystalline lattice imperfections which act as a natural dopant. A popular material, $\mathrm{Bi}_{2} \mathrm{Se}_{3}$, is $n$-doped by the Se vacancies. Along with the attempts to reduce the bulk charge carriers density, experimentalists develop techniques which would allow them to register a separate conduction channel along the surface of a topological insulator. Measurements of the low-field magnetoresistance combined with electrostatic gating of thin-film samples became a burgeoning and promising direction of experiments; an incomplete list of works which appeared within a span of a year and devoted to $\mathrm{Bi}_{2} \mathrm{Se}_{3}$ include Refs. [10-14].

Making sample thinner mitigates the parasitic bulk conductance. Electrostatic gating may help in reducing the bulk density of charge carriers [10, 11, 13]. More importantly, it 
may affect the electron tunneling between the bulk and the putative surface 2D band [13, 14]. Measurement of the low-field magnetoresistance may hold the key in detecting the conduction along a separate surface band.

The low-field anomaly in the magnetoresistance is associated with the interference correction to the Drude conductivity $\sigma_{D}$. At low temperatures, $\sigma_{D}$ is defined by independent acts of scattering of electrons off the imperfections of the crystal and is proportional to the classical electron diffusion constant $D$. If an electron wave preserves its coherence for a sufficiently long time $\tau_{\varphi}(T)$, then the interference between the electron partial waves scattered off different sites affects the conductivity. The sign of the interference correction to conductivity depends on spin-orbit interaction. In its absence, the correction is negative ("weak localization"). Spin-orbit interaction leads to suppression of backscattering, resulting in the weak anti-localization (WAL). Being an electron interference effect, WAL is degraded by a magnetic field $[15,16]$. Its characteristic value $B_{\varphi}$ corresponds to the flux quantum $\Phi_{0}$ piercing a typical trajectory capable to contribute to the interference. The area under such trajectory is $l_{\varphi}^{2} \sim D \tau_{\varphi}$, yielding $B_{\varphi}(T) \sim \Phi_{0} /\left[D \tau_{\varphi}(T)\right]$. For a diffusive $2 \mathrm{D}$ electron system, the resulting magnetoresistance $\Delta \sigma_{\mathrm{WAL}}(B) \equiv \sigma(B)-\sigma(0)=\left(A e^{2} / \pi h\right) \ln \left(B_{\varphi} / B\right)$ at field $B \gg B_{\varphi}$ (here $\ln (\ldots)$ is an asymptote of a known function [15] valid at arbitrary $B / B_{\varphi}$; "weak" in WAL means $\left.\left|\sigma_{W A L}-\sigma_{D}\right| / \sigma_{D} \ll 1\right)$. As long as one deals with a single-component electron system, the coefficient $A$ here is universal, $A=1 / 2$; it is the same for a single-layer $2 \mathrm{D}$ system and for a thin film $[15,16]$ consisting of many atomic layers. The WAL corrections add for systems which are isolated from each other. Having two independent parallel conduction channels would yield $A=1$, regardless the ratio of the Drude conductivities of the two sub-systems.

The relation between $A$ and the number of parallel channels is at the heart of experiments $[13,14]$, and also was touched upon in Ref. [11]. The found $\Delta \sigma(B)$ dependence $[13,14]$ agrees well with the functional form provided by 2D WAL theory [15]. However, the coefficient $A$ depends on the gate voltage $V_{G}$. For some devices $[11,13,14], A$ changes from $A=1 / 2$ all the way to $A=1$.

A very plausible interpretation of that variation is presented in [14]: At zero or positive bias applied to the top gate of their devices (made of $20 \mathrm{~nm}$ thick $\mathrm{Bi}_{2} \mathrm{Se}_{3}$ ) electrons from the $n$-doped bulk reach the surface states easily; the entire film acts as a single electron system, and $A=1 / 2$. At negative bias, the Dirac point of the gapless surface states 
emerges from under the Fermi level, and, in some window of $V_{G}$, a peculiar $p-n$ junction is formed. One side of the junction is the $n$-doped bulk, while the opposite side is the Dirac surface band populated with holes. The depletion region of the junction separates the film in two sub-systems, $A=1$. A stronger negative bias apparently leads to the bulk inversion (accompanied by a precipitous drop in the resistance). Further confirmation of the crossover between the single-system transport and the parallel conduction of the surface and bulk comes from the analysis of the $l_{\varphi}(T)$ dependence [14].

To conclude, we mention here that the samples and data of Refs. [13, 14] look pretty similar, while interpretation is somewhat different: authors of Ref. [13] conclude that at the negative bias conduction occurs along the two surfaces of the film, while its bulk does not contribute to conductivity. Hopefully, future experiments will resolve this issue.

[1] L. Fu, C. L. Kane, and E. J. Mele, Phys. Rev. Lett. 98, 106803 (2007).

[2] J. E. Moore and L. Balents, Phys. Rev. B 75, 121306 (2007).

[3] R. Roy, Phys. Rev. B 79, 195321 (2009).

[4] L. Fu and C. L. Kane, Phys. Rev. B 76, 045302 (2007).

[5] H. Zhang, C.-X. Liu, X.-L. Qi, X. Dai, Z, Fang, and S.-C. Zhang, Nature Physics 5, 438 (2009).

[6] H.-J. Zhang, C.-X. Liu, X.-L. Qi, X.-Y. Deng, X. Dai, S.-C. Zhang, and Z. Fang, Phys. Rev. B 80, 085307 (2009).

[7] M. Z. Hasan, D. Hsieh, Y. Xia, L. A. Wray, S.-Y. Xu, and C. L. Kane, preprint arXiv:1105.0396 (2011).

[8] Z. Alpichshev, J. G. Analytis, J.-H. Chu, I. R. Fisher, Y. L. Chen, Z. X. Shen, A. Fang, and A. Kapitulnik, Phys. Rev. Lett. 104, 016401 (2010).

[9] J. Seo, P. Roushan, H. Beidenkopf, Y. S. Hor, R. J. Cava, and A. Yazdani, Nature 466, 343 (2010).

[10] J. Chen, H. J. Qin, F. Yang, J. Liu, T. Guan, F. M. Qu, G. H. Zhang, J. R. Shi, X. C. Xie, C. L. Yang, K. H. Wu, Y. Q. Li, and L. Lu, Phys. Rev. Lett. 105, 176602 (2010).

[11] J. G. Checkelsky, Y. S. Hor, R. J. Cava, and N. P. Ong, Phys. Rev. Lett. 106, 196801 (2011)

[12] H.-T. He, G. Wang1, T. Zhang1, I.-K. Sou, G. K. L. Wong, and J.-N. Wang, Phys. Rev. Lett. 
106, 166805 (2011).

[13] J. Chen, X. Y. He, K. H. Wu, Z. Q. Ji, L. Lu, J. R. Shi, J. H. Smet, and Y. Q. Li, Phys. Rev. B 83, 241304 (2011).

[14] H. Steinberg, J.-B. Laloö, V. Fatemi, J. S. Moodera, P. Jarillo-Herrero, preprint arXiv:1104.1404 (2011).

[15] S. Hikami, A. I. Larkin, and Y. Nagaoka, Prog. Theor. Phys. 63, 707 (1980).

[16] P. A. Lee and T. V. Ramakrishnan, Rev. Mod. Phys. 57, 287 (1985). 